\title{
Point: Counterpoint synopsis of cardiometabolic risk after spinal cord injury
}

\author{
David R. Gater $\mathbb{D}^{1}$
}

Received: 25 October 2019 / Revised: 30 October 2019 / Accepted: 31 October 2019

(c) International Spinal Cord Society 2019

Sabharwal's Point manuscript "Addressing Cardiometabolic Risk in Adults with Spinal Cord Injury: Acting Now Despite Knowledge Gaps" [1] and Stillman's Counterpoint manuscript "Guideline for the Identification and Management of Cardiometabolic Risk After Spinal Cord Injury: A Case of Unsubstantiated Recommendations" [2] have raised excellent points regarding the recently published guidelines for identification and management of cardiometabolic risks after spinal cord injury (SCI) [3]. Notably, current definitions of obesity used to characterize metabolic syndrome for persons without SCI have been inappropriately applied to persons with SCI due to previously unrecognized disparities in body fat attributed to marked sarcopenia and osteopenia in the latter population. Appropriate adjustments and/or rigorous body composition assessment have unmasked accelerated body fat accumulation in persons with SCI significantly greater than that in able-bodied populations. Adipose tissue secretes multiple proinflammatory cytokines, prothrombotic factors, nonesterified fatty acids, and hormones that mediate insulin resistance, hypertension, dyslipidemia, and arteriosclerosis $[4,5]$.

The biomarkers for diabetes and dyslipidemia in the general population are somewhat nonspecific and yet are used to identify persons at elevated risk who would benefit from intervention. For example, hyperglycemia, hemoglobin A1c, oral glucose tolerance tests, and HOMA-IR indicate elevated plasma glucose but fail to identify true insulin resistance or the time course of the disease relative to pancreatic $\beta$-cell dysfunction. True insulin resistance can only be determined through the complex, labor intensive hyperinsulinemic, euglycemic clamp, or intravenous

David R. Gater

dgater@miami.edu

1 Department of Physical Medicine \& Rehabilitation, Leonard M. Miller School of Medicine, The University of Miami,

Florida, USA glucose tolerance testing. Nonetheless, elevated levels of any of these biomarkers should alert the clinician to begin intervention, including diet, exercise, and potentially pharmacology. Similarly, dyslipidemia characterized by elevated triglycerides and reduced HDL-c in persons with SCI should be aggressively managed through diet, exercise, and pharmacology as adiposity is most likely a major contributor to the condition. Adiposity increases blood pressure through at least four known physiological mechanisms including arterial endothelial damage by proinflammatory cytokines, hepatic synthesis of angiotensinogen, volume expansion through stimulation of the renin-angiotensin-aldosterone system and mechanical compression of the kidneys [5], likely causing the transition from neurogenic hypotension to essential hypertension in persons with chronic SCI; minimally exercise and diet to reduce adiposity is reasonable and prudent.

The guidelines clearly advocate additional research in each of the areas listed above, and randomized, placebocontrolled trials across multiple sites are certainly warranted. Best practices dictate appropriate screening and management of obesity, with appropriate interventional strategies for the comorbidities of diabetes, dyslipidemia, and hypertension employed in this special population. Clinician paralysis, i.e., failure to act in the face of this rising epidemic, is a poor and unnecessary option in managing our patients with SCI.

\section{Compliance with ethical standards}

Conflict of interest The author declares that he has no conflict of interest.

Publisher's note Springer Nature remains neutral with regard to jurisdictional claims in published maps and institutional affiliations. 


\section{References}

1. Sabharwal S. Addressing cardiometabolic risk in adults with spinal cord injury: acting now despite knowledge gaps. Spinal Cord Ser. Cases. 2019. https://doi.org/10.1038/s41394-019-0241-5.

2. Stillman MD, Williams S. Guideline for the identification and management of cardiometabolic risk after spinal cord injury: a case of unsubstantiated recommendations. Spinal Cord Ser. Cases. 2019. https://doi.org/10.1038/s41394-019-0240-6.
3. Nash MS, Groah SL, Gater D, Dyson-Hudson TA, Lieberman JA, Myers J, et al. Identification and management of cardiometabolic risk after spinal cord injury: Clinical Practice Guideline for Health Care Providers. Top Spinal Cord Inj Rehabil. 2018;24:379-423.

4. Farkas GJ, Gater DR. Neurogenic obesity and systemic inflammation following spinal cord injury: a review. J Spinal Cord Med. 2018;41:378-87.

5. Gater D. Pathophysiology of obesity after spinal cord injury. Top Spinal Cord Inj Rehabil. 2007;12:20-34. 